\title{
Detection of Brain Tumor using KNN and LLOYED Clustering
}

\author{
Priyanka Aiwale, Saniya Ansari
}

\begin{abstract}
Today world the brain tumor is life threatening and the main reason for the death. The growth of abnormal cells in brain leads to brain tumor. Brain tumor is categorized into malignant tumor and benign tumor. Malignant is cancerous whereas Benign tumor is non-cancerous. Diagnosing at earlier stage can save the person. It is actually a great challenge to find the brain tumor and classifying its type. Detection of Brain Tumor and the correct analysis of the Tumor structure is difficult task. To overcome the drawbacks of exiting brain tumor detection methods the proposed system is presented using KNN \& LLOYED clustering. Undoubtedly, this saves the time as well as it gives more accurate results as in comparison to manual detection. The proposed method is a novel approach for detection Tumor along with the ability to calculate the area (\%age) occupied by the Tumor in the overall brain cells. Firstly, Tumor regions from an $M R$ image are segmented using an OSTU Algorithm. KNN\& LLOYED are used for detecting as well as distinguishing Tumor affected tissues from the not affected tissues. Total twelve features are extracted like correlation, contrast, energy, homogeneity etc. by performing wavelet transform on the converted gray scale image. For feature extraction DB5 wavelet transform is used.
\end{abstract}

Keywords- KNN\& Lloyd, wavelet transform, tumour, MRI image

\section{INTRODUCTION}

Cerebrum Tumor is one of the real reasons for death among individuals. The manifestations of a mind Tumor rely upon Tumor size, sort and area. Indications might be caused when a Tumor pushes on a nerve or damages a piece of a cerebrum. Additionally, they might be caused when a Tumor obstructs the liquid that moves through and around or when the mind swells since develop of liquid. Cerebral pains, queasiness and heaving, Changes in discourse, vision or hearing, issue adjusting or strolling, changes in temperament, identity or capacity to focus, issues with memory, muscle snapping or tingling, deadness or shivering in the arms or legs. Accurate identification of the type of mind variation among the majority is extremely basic for treatment arranging which could restrict the deadly outcomes. [2] Detection of mind Tumor manually is a recurring activity which consumes a lot of time and also the results are not accurate, shifts starting with one specialist then onto the next. PC supported robotized frameworks provides the appropriate outcomes.

Revised Manuscript Received on February 05, 2020.

* Correspondence Author

Ms. Priyanka Aiwale*, Pursuing M.E.( E \& TC Engineering) in Dr D Y Patil School Of Engineering, Lohegaon Pune

Dr.Saniya Ansari, Associate Professor in Dr. D Y Patil School of Engineering, Lohegaon, Pune

(c) The Authors. Published by Blue Eyes Intelligence Engineering and Sciences Publication (BEIESP). This is an open access article under the CC BY-NC-ND license (http://creativecommons.org/licenses/by-nc-nd/4.0/)
Not only being exactly same, these procedures must scope at a brick pace with a mind set that the final target for their implementation on continuous applications. MRI helps to analyze brain Tumor along with CT images as well as ultrasonic or X-Rays. MRI (Magnetic Resonance Imaging) is an essential instrument utilize in a great many fields of recommendation which is outfitted for producing an explicit image of any part of the body of human. X-ray remains for MRI. A Magnetic Resonance Imaging scanner make use of magnets for the objective of enrapturing as well as for energizing hydrogen cores (single proton) in tissue of humans, that produces a flag that can be distinguished and it's encoded spatially, bringing about images of the body. The MRI machine produces radio recurrence (RF) beat that particularly ties just to hydrogen. The framework sends the beat to that particular territory of the body that should be inspected. Because of the RF beat, protons retain the vitality expected to influence them to turn in an alternate heading. This is implied by the reverberation of MRI. The RF beat influences the protons to turn at the larmour recurrence, in a particular bearing. This recurrence is discovered in light of the specific tissue being imaged and the quality of the principle attractive field. [5]

Grouping of the mind Tumor is likewise a vital undertaking for treatment arranging. There are two sorts of Tumor which are-benevolent (non-destructive) and threatening (carcinogenic) tumors. Ordinary strategies include intrusive systems, for example, biopsy, lumbar cut and flag tap technique, to identify and group cerebrum Tumor into benevolent and harmful which are exceptionally agonizing and tedious. Wavelet investigation is a practicable strategy suitable to unveil various sections of information which other flag as procedures for examination. Segmented the images at a great levels, this method can eliminate much better reason of interest from itself as well as inflates the behavior of the image. To de-noising a flag, equipment is done with no extensive debasement. [7]

\section{RELATED WORK}

In below section, various techniques utilized by various authors are summarized grounded on primary categories such as segmentation, feature extraction as well as classification method used.

Jin Liu, Min Li, Jianxin Wang et al, studies the MRI based brain Tumor segmentation which is more and more attractive because of good soft tissue contrast and noninvasive imaging of Magnetic Resonance. Imaging images. They purposed to make an extensive introduction for MRIbased brain Tumor segmentation strategies. 


\section{Detection of Brain Tumor using KNN and LLOYED Clustering}

Then, the preprocessing activities as well as the state-ofthe-art methods of MRI based Tumor segmentation are actually introduced. [1]

Pavel Dvorak and Bjoern Menze et al, Indeed, even under treatment, patients don't make due all things considered over fourteen weeks after conclusion [3]. Present day medicines incorporate surgery, radiotherapy, chemotherapy or all of them. X-ray is very beneficial to make use of gliomas in various clinical practices, as it is conceivable to procure MRI arrangements giving corresponding information. An actual division of glioma's as well as its intra-tumoural structures is essential for treatment arranging, and also for the regular follow-up schedules. Manual division is laborious and subjected along with intra-rater blunders hard to summarize. In this manner, doctors more often than not utilize harsh measures for assessment. Hence, accurate self-loader or perhaps programmed techniques are needed. [4]

V. Karthikeyan, B. Menze and K. Sreedhar et al, the Tumor mass impact alter the cause of action of the encompassing typical tissues. CNNs have been utilized to win a few question acknowledgment [6], [12] as well as challenges of natural picture division [5]. Since a CNN operates over patches utilizing pieces, it has the benefit of considering as well as being used with crude information. In the arena of mind Tumor division, late proposition additionally examine the utilization of CNNs [11].

J. Selvakumar, A. Lakshami \& T. Arivoli et al, analyzes the methodologies carried out by the image Intensification used in Mathematical Morphological [MM] theory on the dark images. Some Morphological Transformation have been processed through Block Analysis, Morphological Operation and Opening by Reconstruction on the Images with poor lighting. Analysis of the methods which is mentioned above illustrated through the processing of images with various filtering techniques along with various background images of less intensity of light. [7]

Raunaq Rewari, with the utilization of pan morphological methods for the purpose of detection of various background features of the images with poor lighting has implemented the improvement in the digital images. The initial operator works with the information retrieved from the block analysis while the next transformation make use of the reconstruction opening employed to state various backgrounds [8].

Stefan Bauer, Roland Wiest et al, are the creators decided on 2D filters despite the fact that 3D filters can exploit the 3D way of the pictures; however, it builds computational load. The vast spatial and basic fluctuation in mind tumours is additionally an essential worry about utilizing information growth. [9]

K. Sreedhar and B. Panlal, taken automation of brain Tumor segmentation continues to be a challenging task because of significant variations in its structure. In this paper, an automated brain Tumor segmentation algorithm using deep convolutional neural network (DCNN) is presented. [12]

Nikesh T. Gadare, Dr. S. A. Ladhake, et al, implemented few of the transformations which were morphological in nature and these were processed through block analysis, morphological operations followed by reconstruction opening of images with less intensity of light. Through Weber's Law Operator, Background detection and Image enhancement are illustrated. In Mathematical Morphology it has transformation that enables filtering of the Image with new contour leads to closing and opening by reconstruction. [13]

Bjoern Menze and Pavel Dvorak worked on the medical images includes an excessive similarity in the intensities of close by pixels and a powerful correlation of various image modalities. All the images deal with correlation used by local image patches. As well as, there is a high correlation between close labels in the image; this feature is utilized in "local structure prediction" of the "local label patches. For 3D segmentation tasks and for systematically evaluating different parameters that are appropriate for the dense annotation of anatomical" structures, local framework prediction approach are used by them. [14]

Vaishnavi Mehekare, Dr.S.R., Ganorkar, from all among cerebrum tumors, Glioma are the most widely recognized, forceful, prompting a brief long term in their most lofty evaluation. There are different purposes of automatic division strategy in light of Convolutional Neural Networks, investigating little kernel. The use of kernel permits outlining a far more deep design, apart from not having a destructive outcome against over fitting, provided the less number of weights in the system [15].

V. Karthikeyan, B. Menze and K. Sreedhar et al, the Tumor mass impact alter the cause of action of the encompassing typical tissues. CNNs have been utilized to win a few question acknowledgment [6], [12] as well as challenges of natural picture division [5]. Since a CNN operates over patches utilizing pieces, it has the benefit of considering as well as being used with crude information. In the arena of mind Tumor division, late proposition additionally examine the utilization of CNNs [11].

\section{PROPOSED METHODOLOGY}

Image processing techniques are being used to detect the brain tumor. For the purpose of detecting Tumor in the MRI images, MATLAB software is used. The figure shown below is the block diagram of the proposed system.

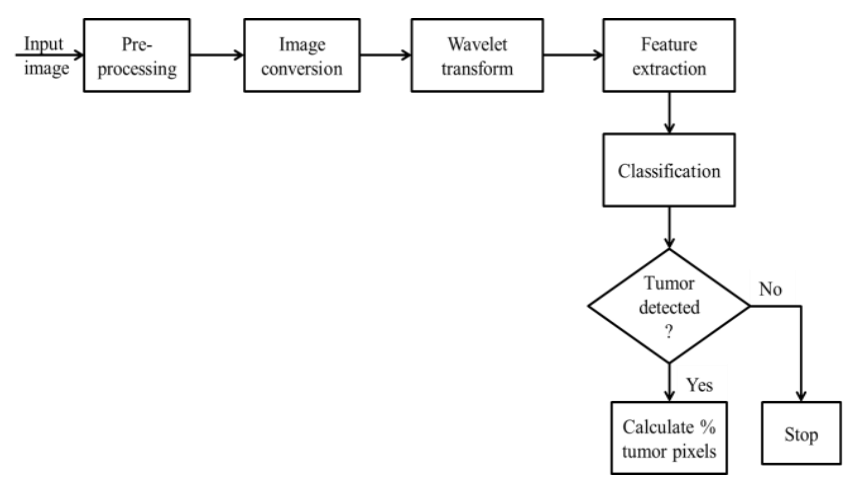

Figure 1: Block Diagram of proposed system 
The detail description of system proposed is as follows:

- Pre-processing: It generally entails removal of background noise having frequency low, 1normalizing the intensity of the individual particles' images, masking 1 of some portions of the images and removing reflections. Image pre-processing is the method to improve data images prior to computational processing.

- Image conversion: In greyscale image or RGB image is that image the value of each pixel is only a single sample which carries information related to the intensity of light or in other words which represents only the amount of light. This sort of images is composed of various shades of gray colour. The range of the contrast from black colour at the weakest intensity to the white colour at the strongest. Keeping this in mind, the conversion of the image in black and white is done. As we understand Tumor is actually big enough to not deemed as tiny bound, therefore we are going to detach little pixel bound.

- Wavelets transform: The Daubechies wavelets, based on each wavelet type of this class, there is a scaling function called the father wavelet which generates an orthogonal multi resolution analysis. The scaling filter associated with the Daubechies wavelet specified by wname. Where $\mathrm{f}$ is a real-valued vector.

- Feature extraction: For the purpose of extracting features from input image different operations are needed to perform like entropy, contrast, correlation, energy, root mean square, standard deviation etc.

- Classification: KNN \& LLOYED are used for the purpose of classifying the tissue 1into normal or cancerous. If the tissue is normal or not-infectious, no Tumor detected displays on MATLAB output window. If the tissue is infectious or if Tumor is detected then the following steps are taken.

Step 1: For smoothing the Tumor MRI Image low pass and high pass filter are applied.

Step 2: For encircling the areas which are affected OSTU Thresholding is used. Draw a circle of maximum possible size covering maximum affected area and next then other circle of small size are drawn.

Step 3: One circle having exact center as that of maximum radius circle from above step with $60 \%$ large radius is chosen so that it can cover complete affected areas called region of interest.

Step 4: For calculating the area of Tumor cells thresholding is performed. Thresholding can be approximated as follow:

$$
\% \text { Area }=\frac{\text { no. of tumour pixels }}{\text { no. of total brain pixels }} \times 100
$$

Step 5:

Segment the tumor

Step 6: Classify the tumor

Step 7: $\quad$ Display the resulting Image

\section{ALGORITHM}

1. Start

2. Take input original MRI brain image

3. Convert it into gray scale

4. Filter the image using LPF \& HPF

5. Morphological operations on image

6. Take OSTU Segmentation

7. LLOYD clustering to segment Tumor

8. Use KNN to find Equlidian distance

9. Hybrid feature extraction using 2 stage Discrete Wavelet Transform

10. Calculate contrast, colleration, Energy, Mean, RMS, Standard Deviation, Smoothness

Tran image using PNN \& RBF

\section{FLOW CHART \& ALGORITHM USED}

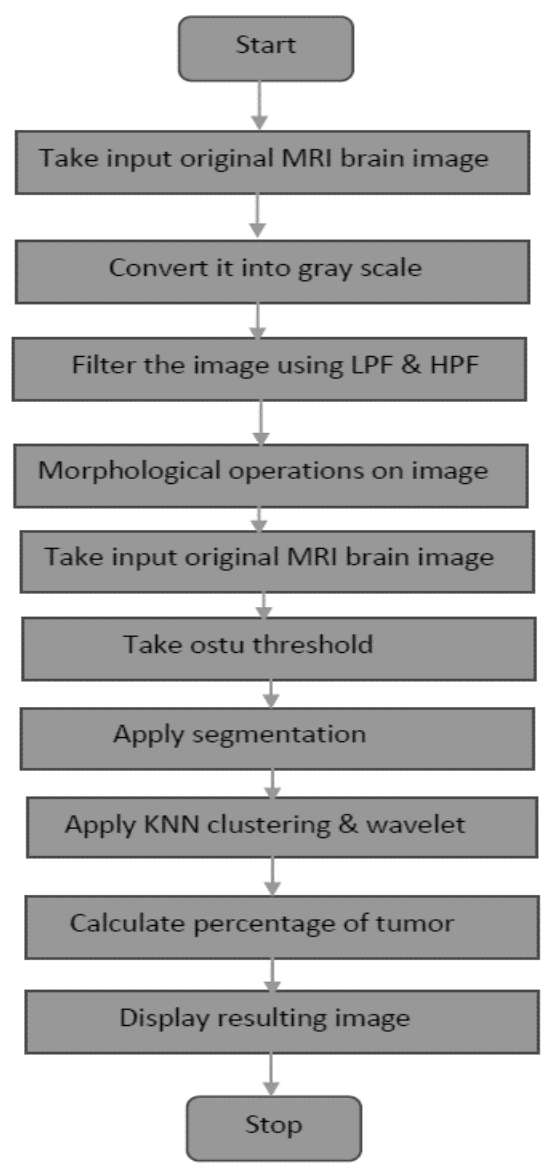

V. RESULT AND DISCUSSION

Table 1. Image Parameters of Feature Extraction

\begin{tabular}{|c|c|}
\hline $\begin{array}{c}\text { Image Feature } \\
\text { Parameter }\end{array}$ & Value \\
\hline Contrast & 4.6787 \\
\hline Correlation & 0.5147 \\
\hline Energy & 0.4659 \\
\hline Homogeneity & 0.8131 \\
\hline Mean & 0.3217 \\
\hline Standard Deviation & 1.457 \\
\hline Entropy & 3.024 \\
\hline RMS & 0.3217 \\
\hline Variance & 1.4588 \\
\hline Smoothness & 0.9992 \\
\hline
\end{tabular}


Table 2 Image Parameters of Recognition Accuracy

Below Figures, shows the output result of all steps used with KNN and LLOYD clustering. These figure shows that all outperforming the existing methods of classification on available dataset images.

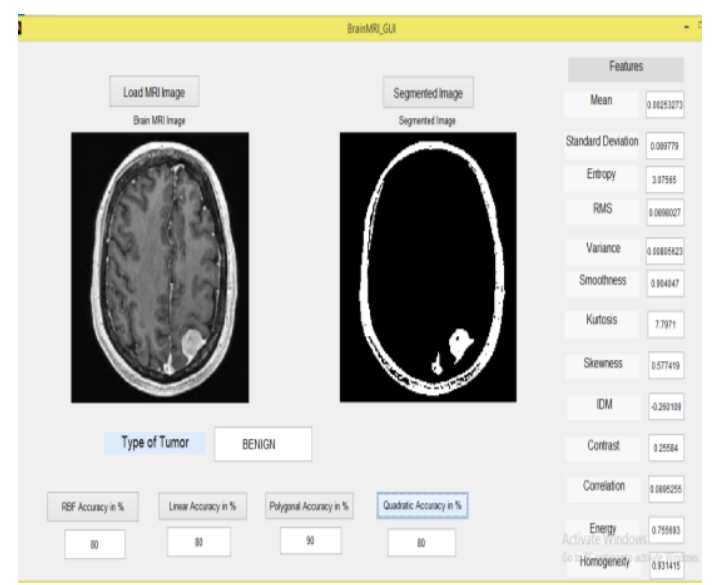

Fig.2 Output of Feature Extraction by using GUI

\section{CONCLUSION}

Detection of Brain Tumor and the correct analysis of the Tumor structure is difficult task. To overcome the drawbacks of exiting brain tumor detection methods the proposed system is presented using KNN \& LLOYED clustering. The proposed method is a novel approach for detection Tumor along with the ability to calculate the area (\%age) occupied by the Tumor in the overall brain cells. Firstly, Tumor regions from an MR image are segmented using an OSTU Algorithm. KNN\& LLOYED are used for detecting as well as distinguishing Tumor affected tissues from the not affected tissues. These algorithms are used to calculate the area occupied by brain tumor. Low pass and High Pass filter along with morphological operation like dilation and erosion are used effectively to remove noise. Total twelve features are extracted like correlation, contrast, energy, homogeneity etc. by performing wavelet transform on the converted gray scale image . For feature extraction DB5 wavelet transform is used. The proposed system calculates the area (\%age) occupied by the Tumor in the overall brain cells.

In future Scope MRI brain Tumor will be classify using CNN \& Deep Learning algorithm to obtain good result of MRI image, it can be possible by using Neural Network.

\section{REFERENCES}

1. Saniya Ansari, Dr U. S Sutar "an efficient method of segmentation for handwriting Devnagri word recognization" international journal of scientific \& engineering research (IJSER) volume 6 issue 5 May 2015 ISSN 2229-5518 pp230-235

2. Saniya Ansari, Dr U. S Sutar "an efficient method of segmentation for handwriting devnagri word recognization" international journal of computer applications ISSN (0975-8887) volume -126 September 2015 edition

3. S. Bauer et al., "A review of x-ray based therapeutic picture examination for mind Tumor thinks about," Physics in solution and science, vol. 58, no. 13, pp. 97- 129, 2013.

4. S'ergio Pereira, Adriano Pinto, Victor Alves and Carlos A. Silva, "Brain Tumor Segmentation utilizing Convolutional Neural Networks in MRI Images",2016.

5. Pavel Dvorak and Bjoern Menze,"Structured Prediction with Convolutional Neural Networks for Multimodal Brain Tumor Segmentation, MICCAI-BRATS 2015.
6. Sheela. V. K and Dr. S. Suresh Babu,"Processing Technique for Brain Tumor Detection and Segmentation," International Research Journal of Engineering and Technology Volume: 02, June-2014

7. Jaypatel and Kaushal Doshi, "An investigation of Segmentation Method for recognition of Tumor in Brain", Advance in Electronic and Electric Engineering, 2014.

8. B. Menze et al., "The multimodal mind Tumor picture division benchmark (whelps)," IEEE Transactions on Medical Imaging, vol. 34, no. 10, pp. 1993-2024, 2015.

9. J. Selvakumar, A. Lakshami \& T. Arivoli,"Brain Tumor Segmentation and Its Area Calculation utilizing K-mean Clustering and Fuzzy CMean Algorithm", IEEE-International Conference On Advances In Engineering, March30,2012.

10. Raunaq Rewari, "Programmed Tumor Segmentation Using Convolutional Neural Network."

11. Stefan Bauer, Roland Wiest and Lutz-P Nolte, "A Survey Of MRIbased restorative picture examination for Brain Tumor Studies".

12. Vaishnavi Dr. P. Eswaran "Enhanced Color Image Enhancement Scheme utilizing Mathematical Morphology ", Volume 3, Issue 4, April 2013 IJARCSSE.

13. V. Karthikeyan1, V. J. Vijayalakshmi, P. Jeyakumar, "A Novel Approach For The Enrichment Of Digital Images Using Morphological Operators", 2013.

14. K.Sreedhar and B.Panlal, Enhancement of images using morphological transformation, 2012.

15. Nikesh T. Gadare, Dr. S. A. Ladhake, Prof. P. D. Gawande, "Mathematical Morphology based Image Enhancement and Background Detection" 2014.

16. Pavel Dvo 'r'ak1,2 and Bjoern Menze3 Structured Prediction with Convolutional Neural Networks for Multimodal Brain Tumor Segmentation, 2015

17. Vaishnavi S. Mehekare, Dr.S.R.Ganorkar, "A Survey on Brain Tumor Detection Using Neural Network 2017.Samjith Raj C.P. and Shreeja $\mathrm{R}$, Automatic brain Tumor tissue detection in T-1 weighted MRI 2017.

18. Manisha, Radhakrishnan.B and Dr. L.Padma Suresh, "Tumor Region Extraction using Edge Detection Method in Brain MRI Images" 2017

19. V. Zeljkovic1, C. Druzgalski2, Y. Zhang1, Z. Zhu1, Z. Xu1, D. Zhang1, P. Mayorga3, "Automatic Brain Tumor Detection and Segmentation in MR Images" 2014.

20. Anatoly Sorokin, Evgeny Zhvansky, Konstantin Bocharov, and Vsevolod Shurkhay, Alexander Potapov, "Multi-label classification of brain Tumor mass spectrometry data" 2017.

21. Alexis Arnaud, Florence Forbes, Nicolas Coquery, Nora Collomb, Benjamin Lemasson, and Emmanuel L. Barbier, "Fully Automatic Lesion Localization and Characterization: Application to Brain Tumours using Multi parametric Quantitative MRI Data"2018.

22. Swathi P S, "Brain Tumor Detection and Classification Using Histogram Thresholding and ANN"2015

23. Ms. Priya Patil, Ms. Seema Pawar, Ms. Sunayna Patil, Prof. Arjun Nichal, "A Review Paper on Brain Tumor Segmentation and Detection"2017.

24. Moitra D and Mandal R" Review of Brain Tumor Detection using Pattern Recognition Techniques"2017

\section{AUTHORS PROFILE}

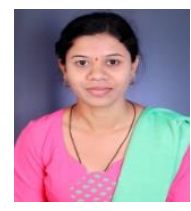

Ms. Priyanka Aiwale, is Pursuing M.E.( E \& TC Engineering) in Dr D Y Patil School Of Engineering, Lohegaon Pune under Savitribai Phule University, Pune

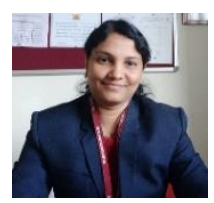

Dr.Saniya Ansari, is working as an Associate Professor in Dr. D Y Patil School of Engineering, Lohegaon, Pune. Her research interest ares are Digital Image Processing, Neural Networks \& Robotiics and automation.

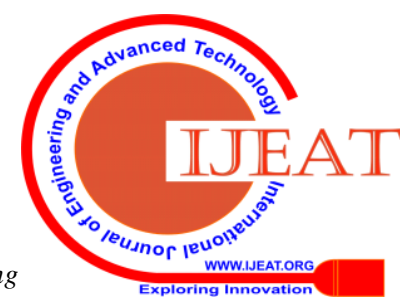

